\title{
Muséologies
}

Les cahiers d'études supérieures

muséologies

\section{Le Louvre Abou Dabi : un nouveau paradigme de gouvernance en émergence?}

\section{Viviane Gautier-Jacquet}

Volume 5, numéro 2, printemps 2011

URI : https://id.erudit.org/iderudit/1033514ar

DOI : https://doi.org/10.7202/1033514ar

Aller au sommaire du numéro

\section{Éditeur(s)}

Association Québécoise de Promotion des Recherches Étudiantes en Muséologie (AQPREM)

\section{ISSN}

1718-5181 (imprimé)

1929-7815 (numérique)

Découvrir la revue

Citer cet article

Gautier-Jacquet, V. (2011). Le Louvre Abou Dabi : un nouveau paradigme de gouvernance en émergence ? Muséologies, 5(2), 12-41.

https://doi.org/10.7202/1033514ar
Résumé de l'article

L'entente historique de coopération culturelle sur 30 ans entre Le Louvre et le gouvernement des Émirats arabes unis signée en 2007 offre à la France l'occasion d'étendre son ascendance culturelle. Si l'Accord a suscité la controverse auprès de certains spécialistes français, il reste que, pour le gouvernement français, la réalisation du Louvre Abou Dabi représente l'occasion de conserver l'équilibre budgétaire de son portefeuille culturel tout en haussant sa rentabilité et en s'assurant une prédominance géoculturelle. En analysant certains des aspects majeurs de cette entente, Viviane

Gautier-Jacquet tente de cerner la signification idéologique de cet accord. 
Viviane Gautier-Jacquet

Le Louvre Abou Dabi : un nouveau paradigme de gouvernance en émergence?

L'entente historique de coopération culturelle sur 30 ans entre Le Louvre et le gouvernement des Émirats arabes unis signée en 2007 offre à la France l'occasion d'étendre son ascendance culturelle. Si l'Accord a suscité la controverse auprès de certains spécialistes français, il reste que, pour le gouvernement français, la réalisation du Louvre Abou Dabi représente l'occasion de conserver l'équilibre budgétaire de son portefeuille culturel tout en haussant sa rentabilité et en s'assurant une prédominance géoculturelle. En analysant certains des aspects majeurs de cette entente, Viviane GautierJacquet tente de cerner la signification idéologique de cet accord.

Viviane Gautier-Jacquet est titulaire d'une Maîtrise ès arts (M.A.) en Histoire de l'art de l'Université de Montréal. Elle poursuit actuellement ses études doctorales. Le présent article est extrait et adapté de son Mémoire de Maîtrise "Le Louvre des sables: le dossier Abou Dhabi ", déposé en 2010.

viviane.gautier@umontreal.ca 


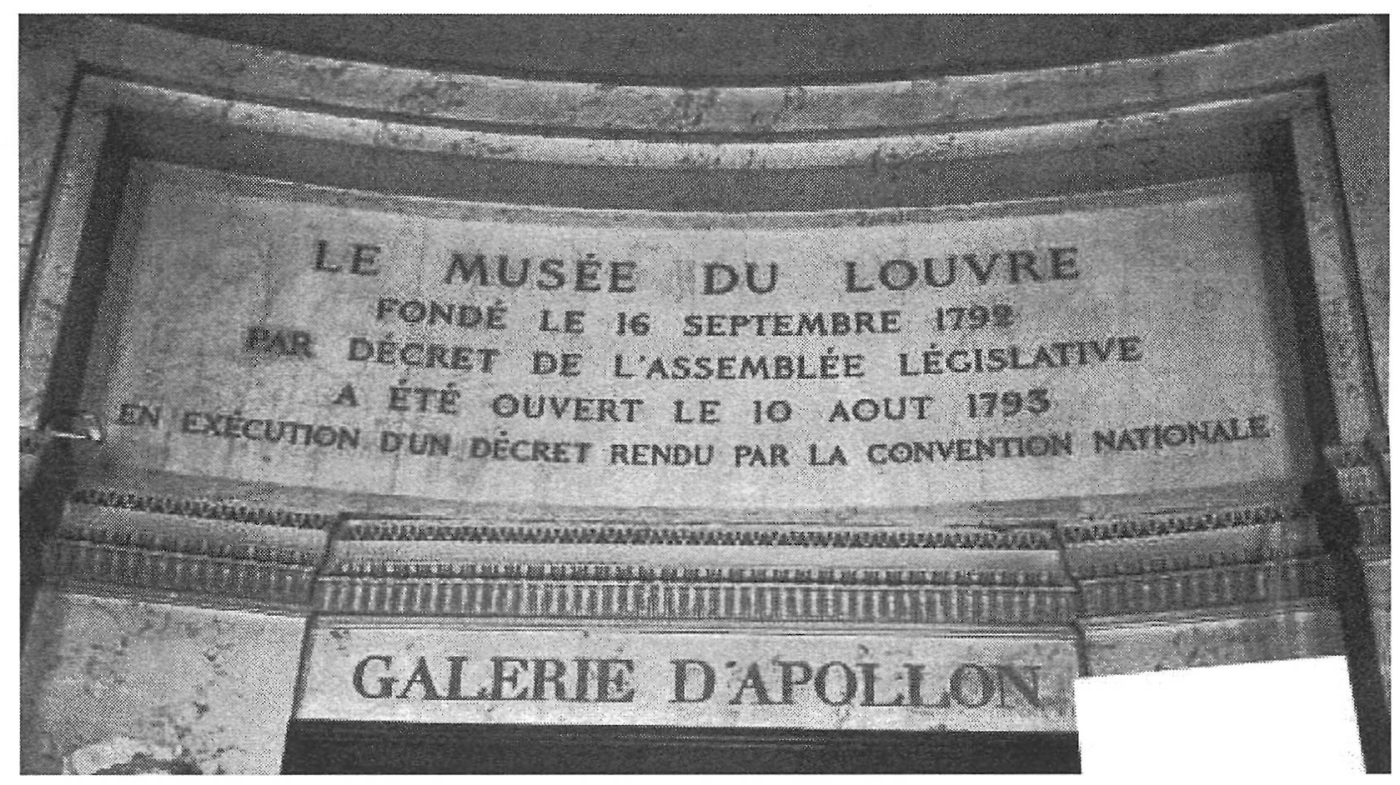

Inscription sur la porte d'entrée de la galerie d'Apollon.

Photo: Viviane Gautier-Jacquet 
A noter qu'il y a différentes façons d'écrire Abou Dhabi; nous respectons ici la graphie utilisée par le musée (nom officiel: Louvre Abou Dabi) et aussi celle utilisée par les personnes que nous citons. 2

Voir dossier de presse sur le site de I'ACENCE FRANCE-MUSÉUMS, laquelle est chargée d'élaborer le projet scientifique et de fournir les prestations de l'accord. <http:// www.agencefrancemuseums.fr/ $\mathrm{fr} /$ ressources-documentaires/> (consulté en janvier 2011).

\section{L'Accord}

Le 6 mars 2007, Renaud Donnedieu de Vabres, ministre de la Culture de la présidence de Jacques Chirac, prononce un discours devant les autorités émiriennes réunies au palais des émirats d'Abou Dhabi': "Nous signons aujourd'hui un accord historique de coopération culturelle entre les Émirats arabes unis et la France, en vue de la création par l'émirat d'Abou Dabi d'un musée à vocation universelle, qui portera le nom de Louvre Abou Dabi ". Jacques Chirac, quant à lui, envoie un message personnel au sheikh Khalifa bin Zayed al Nahyan, président de la Fédération des Émirats arabes unis, qu'il amorce ainsi : "Nous célébrons aujourd'hui un évènement qui fera date; pas seulement pour nos deux pays, dont les relations sont si amicales et si confiantes, mais pour une certaine idée du monde, que les Émirats arabes unis et la France veulent porter ensemble "².

L'accord est en effet historique. Les Émirats arabes unis demandent et reçoivent le concours du musée du Louvre pour la conception et la réalisation du premier musée "universel" hors Occident. Pour ce faire, les autorités tutélaires du Musée national du Grand Louvre, avec la collaboration d'Henri Loyrette, président-directeur général de l'institution, octroient à Abou Dhabi le droit d'utiliser le nom "Louvre" dans le titre du musée émirien, pour une durée de trente ans et six mois, au coût de 400 millions d'euros. L'entente implique également l'envoi périodique, pendant dix ans, d'objets faisant partie des collections du musée parisien et des musées français publics volontaires : 300 œuvres seront d'abord prêtées, pour six mois à deux ans, terme éventuellement renouvelable, puis 250 à partir de la quatrième année et 200 de la septième année à l'expiration de la dixième. Les compensations financières sont fixées à 190 millions d'euros pour le prêt d'œuvres. Par ailleurs, et moyennant 195 millions d'euros, est prévue, sur quinze ans, l'organisation de quatre expositions temporaires annuelles qui seront assemblées à partir des collections françaises. Finalement, l'assistance fournie à la partie émirienne, y compris, entre autres, la formation de spécialistes de l'art et du musée (une antenne de la Sorbonne 
ouverte en 2006 à Abou Dhabi participe à la formation du personnel qualifié du futur musée émirien grâce à un programme d'enseignement qui permet l'accès à un master en histoire de l'art et métiers des musées ${ }^{3}$ ), l'aide des conservateurs français à l'acquisition des œuvres de la future collection permanente d'Abou Dhabi, entre autres, fera l'objet d'une rémunération globale de 165 millions d'euros 4 . Sur le plan financier, le projet rapportera à la France, plus précisément au Louvre et dans une moindre mesure aux musées prêteurs, la somme de 975 millions d'euros, somme incluant 25 millions d'euros versés à titre de mécénat un mois après la signature du contrat ${ }^{5}$.

L'Agence France-Muséums est spécialement créée pour accomplir les modalités du contrat et, en particulier, pour définir le projet scientifique et culturel ainsi que la programmation des œuvres. Elle est constituée d'une douzaine d'établissements publics culturels parmi lesquels figurent le musée du Louvre, le Centre Pompidou, le musée d'Orsay, la Bibliothèque nationale de France, le musée du quai Branly, la Réunion des musées nationaux ${ }^{6}$. Marc Ladreit de Lacharrière, fondateur et directeur de la société holding Fimalac, grand mécène du Louvre, est président du conseil d'administration de France-Muséums, tandis qu'Henri Loyrette est président du conseil scientifique. L'agence a notamment mis en place la Commission de sélection des œuvres en partance pour Abou Dhabi et la Commission des acquisitions pour le compte du futur musée émirien. A ce jour, seule une première liste de 19 œuvres acquises par la commission est publiée et accessible sur le site de l'Agence France-Muséums.

\section{La polémique}

Au-delà de l'aspect mercantile de l'accord, alors que les sommes perçues par la partie française sont considérées, selon les observateurs, fabuleuses ou insignifiantes en comparaison de la valeur des œuvres prêtées, nombreux et déterminants sont les sujets de débat, portant sur l'évolution du musée et sa gestion, sur le défi des institutions culturelles qui, bien qu'à but non lucratif, sont soumises à
3

HULLO-POUYAT, Céline. "Le Louvre à Abu Dhabi ou la quête du cosmopolitismen. In. ROLLAND, Anne-Solène et Hanna MURAUSKAYA (dir.). De nouveaux modèles de musées? Formes et enjeux des créations et rénovations de musées en Europe XIXe $-X X \mid{ }^{e}$ siècles. Paris : L'Harmattan, 2008, p. 293-314. Voir également le site de PARISSORBONNEABOU DHABI (PSUAD): <http://www.sorbonne.ae/sites/fr/ Pages/psuad.aspx> (consulté en avril 2011).

4

Voir à propos de cet accord signé le 6 mars 2007: Journal officiel de la République française. Ministère des Affaires étrangères et européennes. Décret n' 2008-879. <http://www. agencefrancemuseums.fr/fichier/s paragraphe/897/paragraphe_file_1_ en_decret_portant_publiction_de_I accord_intergouvernemental.pdf> (consulté en janvier 2011). 5

Pour des détails concernant l'aspect financier de l'accord, voir COB, André. "Et si on parlait d'argent?". In. Le musée, une institution dépassée? Paris: Armand Colin, 2010.

6

Voir liste complète sur le site de l'ACENCE FRANCEMUSEUMS: <http://Www. agencefrancemuseums. $\mathrm{fr} / \mathrm{fr} / \mathrm{I}$ agence-france-museums/l-agencefrance-museums/> (consulté en avril 2011) 
7

En ce qui concerne la polémique, voir les livres: CLAIR, Jean.

Malaise dans les musées. Paris: Flammarion, 2007; et RYKNER, Didier. Le spleen d'Apollon. Musées, fric et mondialisation. Paris: Nicolas Chaudun, 2008. En ce qui concerne les articles, voir entre autres: CACHIN, Françoise, Jean CLAIR et Roland RECHT. "Les musées ne sont pas à vendre". Le Monde, 12 décembre 2006; LACLOTTE, Michel. "Un Louvre à Abou Dhabi, ce n'est pas si simple». Libération, 29 janvier 2007; NOCE, Vincent. "Les risques du Louvre à Abou Dhabi. 39 conservateurs ont adressé une note au ministre de la Culture". Libération, 3 février 2007; BENHAMOU, Françoise. "Le Louvre d'Abou Dhabi : dévoyé ou rayonnant?». Esprit, n³34, mai 2007. Par ailleurs, rappelons que le site Internet de La Tribune de l'art, élaboré par Didier RYKNER, a mis en place, en son temps, une pétition qui a recueilli environ 5000 signatures de personnalités du monde professionnel de l'art, du musée et de la politique, qui se sont positionnés contre le projet Louvre Abou Dabi. la mondialisation de l'économie et à l'évolution du marché de l'art et de la culture. Ainsi, l'entente intergouvernementale est décriée par nombre d'observateurs et de spécialistes qui considèrent que le projet est motivé par des raisons diplomatiques et économiques, imposé sans négociations publiques et sans projet scientifique préalables à la signature, et qu'il enfreint alors l'éthique de la profession. Les polémistes s'objectent à la commercialisation du vocable historique "Louvre", ainsi qu'à la commercialisation des $œ u v r e s d u$ patrimoine national. Ils dénoncent, en outre, l'atteinte au sens des collections et à la sécurité des œuvres, tandis que le principe de permanence et la vocation à l'exhaustivité des collections sont questionnés. Le conflit d'intérêt des professionnels du Louvre est également contesté ; ceux-ci doivent agir en tant que conseillers à l'acquisition pour un musée étranger alors qu'ils sont agents de l'État français. Toujours selon l'accord, la France prête des œuvres "issues de différents musées publics volontaires"; pour nombre de sceptiques, l'expression est désinvolte car les pressions sur les conservateurs des musées de province peuvent être fortes, ceux-ci risquant d'avoir du mal à résister aux demandes de leur tutelle, éventuellement favorable à l'envoi d'œuvres pour des raisons pécuniaires. Par ailleurs, les adversaires du projet rappellent que la mission du Louvre est historiquement de collaborer avec les musées de province, véritables antennes du musée central, et réprouvent les difficultés chroniques des musées provinciaux à obtenir des prêts, gratuits, du Louvre, suggérant que ceux-ci seront d'autant plus difficiles à recevoir. La question de la censure est également soulevée car le choix des œuvres en partance pour Abou Dhabi est soumis à validation par une commission sur laquelle l'émirat a droit de veto. Finalement, certains observateurs craignent jusqu'à la remise en question - ou au détournement - du principe de l'inaliénabilité des collections publiques françaises'.

Quelle est la signification idéologique de l'Accord du 6 mars 2007 ? Est-ce à dire que de nouveaux paradigmes se mettent en place, avec l'implantation, temporaire de longue durée, du Louvre Abou Dabi ? Dans le présent article, l'analyse porte sur la création politique du Louvre, son 
élaboration, son développement, son rôle diplomatique et sa dépendance financière. La réflexion aborde le concept de musée "universel" ainsi que le statut de patrimoine national et tend à souligner que le musée, depuis sa création à la fin du XVIII e siècle, est un appareil de l'État, que son autonomie institutionnelle, financière, intellectuelle n'a jamais été envisagée. Même s'il a acquis une certaine indépendance vis-à-vis du gouvernement, il est désormais également engagé vis-à-vis de ses mécènes et de l'économie de la culture mondiale dans laquelle il s'inscrit et reste tributaire.

Le cadre théorique de cet essai s'appuie, entre autres, sur les travaux de Dominique Poulot, d'Andrew McClellan et de Tony Bennett, qui ont démontré la dimension construite de la notion de patrimoine national et la nature politique du Louvre depuis sa création. En ce qui concerne l'observation de l'évolution de la gestion des musées, l'ouvrage de Jean-Michel Tobelem ${ }^{8}$, qui analyse les cadres politique, social et culturel, économique et financier dans lesquels les musées français s'insèrent et évoluent, et desquels ils sont tributaires, a été maintes fois consulté. Le corpus étudié ne comprend que de la documentation écrite, constituée de la presse d'actualité, de la presse spécialisée, d'entretiens accordés à ces dernières par des professionnels de la politique culturelle, du musée et de l'histoire de l'art, ainsi que des ressources électroniques, tels le site Internet de $\mathrm{La}$ Tribune de l'art qui se positionne en tête du mouvement de contestation ou le site de l'Agence France-Muséums chargée de développer le projet Louvre Abou Dabi et de fournir les prestations prévues dans l'accord.

\section{Le musée du Louvre : œuvre politique}

Le musée du Louvre est créé à la fin du XVIII siècle. Il est le résultat d'un décret de la Convention nationale, mais la conception du projet revient à la monarchie, qui cherche à apaiser les diverses critiques, de plus en plus virulentes, faites au régime. C'est ainsi que Charles-Claude Flahaut de la Billarderie, comte d'Angiviller, dernier directeur général des Bâtiments du roi, chargé de transformer

\section{8}

TOBELEM, Jean-Michel. Le nouvel âge des musées. Les institutions culturelles au défi de la gestion. Paris: Armand Colin, 2005. 
9

MCCLELLAN, Andrew. Inventing the Louvre: Art, Politics, and the Origins of the Modern Museum in EighteenthCentury Paris. Berkeley (CA), LoS Angeles, Londres: University of California Press, 1994.

10

DUNCAN, Carol et Allan WALLACH. "The Universal Survey Museum ". In. CARBONELL, Bettina Messias (dir.). Museum Studies, An Anthology of Contexts. Malden (MA): Blackwell Publishing, 2004, p. 58. la Grande Galerie du Louvre en un lieu d'exposition de la collection royale, veut créer un muséum à la gloire du roi, du royaume, de la supériorité de l'École artistique française. Pour ce faire, il complète la collection royale par l'achat de quelque 200 œuvres, fait réaliser de nombreux tableaux d'histoire et des sculptures de "Grands Hommes" qui honorent l'histoire de France. Andrew Mcclellan le précise, ces œuvres, spécifiquement réalisées pour le futur public du musée, sont destinées à inspirer des sentiments patriotiques chez les sujets du roi et à impressionner les visiteurs étrangers, tandis que les collections royales sont transformées en arsenal politique ayant pour fonction de manipuler l'opinion 9. La Révolution coupe court au projet de la monarchie; les membres de l'Assemblée nationale reprennent le projet à leur compte, et le Muséum Français est inauguré le 10 août 1793 lors des célébrations du premier anniversaire de la chute de la royauté. Le Louvre devient alors l'instrument stratégique du nouveau pouvoir. Non seulement une partie du palais des rois devient musée public, mais les collections royales qui constituent le noyau du musée deviennent patrimoine national. S'y ajoutent les biens du clergé confisqués par les révolutionnaires ainsi que ceux de la noblesse condamnée ou émigrée ; puis s'ajoutent encore, de manière méthodique, le butin des conquêtes militaires napoléoniennes. Le Concordat de 1802, la Restauration en 1815, font restituer la majorité des œuvres à l'Église et aux pays alliés de la monarchie. Les collections sont cependant vite réapprovisionnées, grâce à l'attention portée aux œuvres nationales et aux judicieuses politiques d'acquisition, faisant du musée du Louvre l'indéfectible symbole de la démocratie culturelle et le gardien du patrimoine national. Sans conteste, la Révolution transforme la notion de public, née au XVIII siècle, notamment avec les expositions du Salon des artistes, car, tel que Carol Duncan et Allan Wallach le soulignent, le visiteur, qui était "sujet du roi", devient "citoyen français", acteur de la politique en place et en quelque sorte "actionnaire" du patrimoine de l'État, tandis que celui-ci devient sujet et objet du musée'º. 
En conséquence, depuis son origine et tout au long du $\mathrm{XIX}^{\mathrm{e}}$ siècle, le musée sert à promouvoir la fierté nationale et contribue à l'élaboration de l'unité collective par le biais du concept de patrimoine national qui lui est inhérent. D'une part, le patrimoine, devenant l'affaire de chacun en devenant l'héritage commun et le témoin de l'histoire de la nation, participe à la construction identitaire de la France postrévolutionnaire, républicaine et laïque. D'autre part, Tony Bennett le montre, le musée sert à uniformiser les comportements sociaux des différentes classes sociales, sur le modèle de la nouvelle élite bourgeoise, en instaurant des autorisations (de parler, mais à voix basse), des interdictions (de bousculer par exemple)". De la même manière, le choix pondéré des œuvres, la scénographie muséale, la présentation chronologique et par écoles nationales servent le discours politique et mettent en avant l'idée de progrès de l'art, de progrès de l'histoire de France. Ce programme d'éducation et d'instruction civique incite les administrateurs du musée à opter pour la gratuité du musée afin que l'édification programmatique soit accessible au plus grand nombre.

Les œuvres qui constituent le patrimoine national sont donc retirées de leur contexte culturel et fonctionnel pour prendre un statut d'œuvres d'art à valeur pédagogique, non seulement sur le plan esthétique, mais aussi sur les plans historique et culturel. Le patrimoine de la nation est ainsi le résultat d'une suite de dispositifs discursifs mis en scène, de prises de position, le résultat délibéré de choix et de renoncements de l'élite intellectuelle qui administre le musée et qui est en lien étroit avec le pouvoir central. Dominique Poulot définit le patrimoine comme étant "transmis, c'est-à-dire glosé, restitué, raconté, travaillé "12. Le Louvre est donc un lieu de discours, un lieu de production de l'histoire, de production de l'identité nationale et, par voie de conséquence, de l'altérité. Il sert à la construction d'une d'histoire et d'une mémoire communes; il participe à la fabrication de la cohésion culturelle nationale. Museum. History, Theory, Politics. Londres et New York: Routledge, 1995.

POULOT, Dominique. Patrimoine et musées. L'institution de la culture. Paris: Hachette, 2001, p. 4. 
13

En particulier, la loi renforce la décentralisation, uniformise le statut des musées de France, définit leur projet culturel et scientifique, limite le contrôle de l'Etat aux musées labélisés "Musée de France". Voir le texte de loi accessible sur le site de LECIFRANCE: <http://www.legifrance.gouv.fr/ affichTexte. do; $j$ sessionid $=8 C A 6662 \mathrm{~A}$ 18EDC516CFFF30D66A840A9D.tpdjo OوV_3?cidTexte=LEGITEXTO0000563 2040GdateTexte $=20110325$ > (consulté en avril 2011).

14

La modalité essentielle de cette loi est de faciliter le mécénat privé. Texte de loi accessible sur le site de LEGIFRANCE : <http://www.legifrance.gouv.fr/affichTexte.do?cidTex te=JORFTEXTOO०000791289GdateTe xte $=>$ (consulté en avril 2011).

15

La Réunion des musées nationaux (RMN) avait jusqu'alors la compétence en matière d'acquisition et percevait, à ce titre, les recettes de la billetterie. Elle englobait ainsi plus d'une trentaine de musées nationaux. Ceux-ci devenant peu à peu des établissements publics, la RMN se retrouve, actuellement, en cours de redéfinition.

\section{Le Musée national du Grand Louvre et sa double dépendance financière}

Le musée de la seconde moitié du XXe siècle se modernise, se démocratise, et l'intérêt, qui était plutôt centré sur les collections, se tourne alors vers le public. Le Grand Louvre, projet d'agrandissement, de rénovation, de réaménagement du palais, inaugure l'ère nouvelle du musée symbolisée aujourd'hui par la pyramide de l'architecte sino-américain leoh Ming Pei. Le rôle statutaire de l'institution s'est également transformé au cours des vingt dernières années. En 1992, le Louvre devient un établissement public, c'està-dire une personne morale de droit public, financée par des fonds publics, avec la mission de servir le public. Les nouvelles dispositions administratives et financières accordées au musée lui permettent de gérer plus librement les ressources allouées par l'État et, par là même, de devenir responsable de ses éventuels déficits. Les lois "Musées " ${ }^{3}$ de 2002 et "Mécénat" 14 de 2003 viennent ajouter de nouvelles dispositions émancipatrices; la nouvelle charte du Louvre l'autorise à solliciter des crédits supplémentaires d'origine privée et lui accorde la compétence, non partagée, en matière d'acquisition's. Quatre grandes sources de financement alimentent aujourd'hui ses revenus: les subventions de l'État; le mécénat et le parrainage; ses recettes propres provenant de la billetterie, des revenus de placements financiers et des activités annexes telles la location d'espaces, les boutiques, la restauration; le fonds de dotation dont le musée est titulaire depuis 2009. Par ailleurs, c'est en 2003, dans le cadre de la nouvelle Loi organique relative aux lois de finances (LOLF), laquelle supervise la performance de la gestion des fonds publics et la transparence de l'information budgétaire, que le ministère de la Culture et l'Établissement public du Louvre signent le premier Contrat d'objectifs et de moyens (COM) qui met en place les orientations gouvernementales sur trois ans. Le contrat vise essentiellement à la rentabilité des crédits octroyés. En substance, trois grandes directives sont énoncées: il faut intensifier l'action culturelle afin d'assurer la haute fréquentation du musée par le public national; il faut mettre en valeur le patrimoine afin de développer la 
coopération avec les musées de région et intensifier le rôle du Louvre à l'international; il faut redéfinir les fonctions professionnelles afin d'optimiser les ressources humaines, techniques et financières ${ }^{16}$. Dans un communiqué de presse du 17 avril 2003, jour de la signature du premier COM, le ministre de la Culture Jean-Jacques Aillagon précise : «Le Louvre s'engage à accroître ses ressources propres de 8,2 millions d'euros sur trois ans et à financer grâce au mécénat l'aménagement de nouvelles salles (20 millions d'euros pour la période 2003/2005, soit un doublement de ses recettes à ce titre)". La création du Louvre Abou Dabi est donc dans la lignée des orientations gouvernementales, notamment en ce qui concerne deux objectifs : l'accroissement de l'implication du Louvre à l'international et le financement par le mécénat. La progression constante des recettes propres du Louvre fait proportionnellement diminuer la subvention étatique qui est de l'ordre désormais de $50 \%$ du budget total du musée. Le Louvre est donc aujourd'hui en état de double dépendance, vis-à-vis de l'État dont il est redevable et vis-à-vis de ses partenaires qui participent de plus en plus à son financement.

\section{Dialogue économique ou dialogue de l'art?}

Les spéculations du marché de l'art qui s'universalise, l'économie de la culture, la marche de la mondialisation participent à la transformation des pratiques muséales. Le partenariat controversé avec le High Museum d'Atlanta ", le lancement du projet d'une succursale à Lens dont l'ouverture est prévue pour 2012 et la création du Louvre Abou Dabi, pour ne mentionner que les activités extra-muros récentes du Louvre, témoignent d'une gestion muséale orientée vers l'expansion géographique d'ordre touristique qui entre en concurrence avec le commerce des loisirs. Ainsi, les œuvres du passé, hébergées dans un musée au nom illustre, bénéficient de prestige que les institutions instrumentalisent à des fins de rentabilité économique. Régis Debray reprend la théorie des signes de Charles Peirce pour montrer que la valeur culturelle de l'œuvre religieuse, le rapport à "l'image-indice" d'une présence mystérieuse, disparaît au profit de «l'œuvre-icône» à valeur esthétique,
16

KREBS, Anne et Bruno MARESCA. Le renouveau des musées. Paris: La Documentation française, coll. "Problèmes économiques et sociaux", 2005. 
17

Voir notamment l'article de RYKNER, Didier. "Le Baldassare Castiglione de Raphaël et Et in Arcadia Ego de Poussin prêtés un an à Atlanta ", posté le 20 janvier 2006 sur le site de La Tribune de l'Art: <http://www.latribunedelart.com/ le-i-baldassare-castiglione-i-deraphael-et-i-et-in-arcadia-ego-i-depoussin-pretes-un-an-a-atlantaarticleoog7ı.html> (consulté en avril 2011).

18

DEBRAY, Régis. Vie et mort de l'image. Paris: Gallimard, coll. "Folio essais", 1992. source de plaisir, de savoir, d'intérêt moral et intellectuel. L'œuvre-icône disparaît à son tour au profit de "l'imagesymbole». Celle-ci a une valeur socioculturelle arbitraire qui mise, notamment, sur l'authentique, l'unique, l'ancien. Debray redéfinit quelque peu ces trois régimes de temporalité des images : le premier régime, le "régime des idoles ", commence avec l'écriture et s'arrête avec l'imprimerie, lorsque commence le "régime de l'art" qui s'étend jusqu'à la venue de la télévision couleurs, laquelle propulse alors le "régime du visuel ". Selon cet auteur, le premier régime se situe dans un temps immobile, son espace est ethnique et il est du ressort du religieux, de la piété ; l'art vient "d'en haut"; il est "solennel ". La deuxième ère se situe dans un temps lent, dans l'espace occidental, et elle est de nature esthétique; l'œuvre vient du génie de l'artiste, "du dedans" ; l'art est illusion, et il est "sérieux". La troisième ère est happée par la vitesse, se propage à l'échelle mondiale et sa nature est d'ordre économique ; l'art vient "du dehors", du marketing par exemple; il est ironique et objet d'expérimentations multiples ə Pour Debray, l'art d'abord assujetti au religieux, puis à la politique des mécènes détenteurs du pouvoir clérical ou politique, est désormais sous l'influence de l'économie, des marchands, des collectionneurs, des commanditaires et du réseau de la diffusion ${ }^{18}$. L'entente franco-émirienne mise certes sur le passé illustre du palais, du musée du Louvre, sur son aura et son pouvoir d'attractivité qui ont une valeur sociale arbitraire, symbole de culture, de prestige d'une société, qui est source d'intérêt économique, financier et diplomatique.

L'Accord Louvre Abou Dabi, signé en mars 2007, prévoit, à l'article $1^{\text {er }}$, la création d'un musée universel "destiné à œuvrer au dialogue entre l'Orient et l'Occident, chaque partie respectant les valeurs culturelles de l'autre». Or, pour les détracteurs de l'accord, la location du nom "Louvre" et des œuvres équivaut à une commercialisation du patrimoine national. En effet, tout porte à croire que les motivations premières soient commerciales:

- Les intérêts pour les Émirats arabes unis. Ils constituent la troisième puissance économique du Moyen-Orient derrière 
l'Arabie saoudite et l'Iran. Leur économie dépend des ressources fossiles non renouvelables et des revenus de leurs investissements financiers qui seraient, à eux seuls, suffisants pour équilibrer les dépenses de l'État. Les autorités cherchent toutefois à diversifier leurs sources de revenus en investissant dans des participations industrielles et dans le secteur des services, dont le tourisme de luxe, avec notamment la création d'un centre culturel sur l'île de Saadiyat. Cette île de 27 kilomètres carrés est à 500 mètres du rivage ; elle sera reliée par deux autoroutes à deux fois cinq voies. Elle se développe en plusieurs phases et son achèvement est prévu pour 2018. Abou Dhabi, misant sur sa situation géographique privilégiée, sorte de pont entre l'Orient et l'Occident, espère recevoir dès 2015 trois millions de voyageurs. Le coût total du projet est estimé à 30 milliards de dollars américains. Il inclut cinq musées : le futur musée universel portant le nom du Louvre réalisé par l'architecte Jean Nouvel, un musée Guggenheim conçu par l'architecte Frank Gehry, un musée maritime produit par Tadao Ando, un centre des arts de la scène réalisé par Zaha Hadid et le musée du patrimoine du sheikh Zayed bin Sultan al Nahyan (père fondateur de la nation) produit par Norman Foster. S'ajouteront à ces musées un parc biennal comprenant 19 pavillons, des salles de concerts, de spectacles, un parc naturel, trois marinas, deux terrains de golf, un circuit de formule 1, une annexe de l'Université de New York, 29 hôtels de luxe dont un de sept étoiles, 8000 villas, etc. Saadiyat, destinée à être un sanctuaire de la culture universelle, lieu de dialogue et d'échanges, sera, avant tout, une destination touristique de première classe, même si les buts avoués du sheikh Mohammed bin Zayed al Nahyan, gouverneur d'Abou Dhabi, sont de faire de la capitale de son pays la capitale culturelle du Moyen-Orient, de renouveler l'art islamique, de renouer avec la grande époque de la culture arabe et de faire des Emirats la plaque tournante des échanges entre l'Orient et l'Occident. Selon l'émir, le but de l'île de Saadiyat doit être de créer un atout culturel pour le monde - une porte d'entrée et un phare pour l'expérience et l'échange culturels. La culture traverse toutes les frontières, ainsi Saadiyat appartiendra au peuple des EAU, à l'ensemble du Moyen-Orient et au reste du monde' ${ }^{19}$.

- Les intérêts pour la France. Les Émirats arabes unis sont le premier partenaire commercial au Moyen-Orient, en l'occurrence grâce au commerce de l'énergie nucléaire, de l'aéronautique militaire et commerciale. La coopération culturelle, scientifique et technique est également présente, en
19

Cité dans SELICSON, Joelle. "Can Museums Buy Happiness?». Museum News, novembre-décembre 2008 , p. 46-53 et 83: "The aim of Saadiyat Island must be to create a cultural asset for the world-a gateway and beacon for cultural experience and exchange [...] Culture crosses all boundaries, and therefore Saadiyat will belong to the people of the UAE, the greater Middle East and the world at large." 
20

La France essuie cependant de gros revers commerciaux aux EAU, notamment en ce qui concerne un important projet nucléaire de 20 milliards de dollars, qui lui a échappé, et elle n'a toujours pas réussi à vendre un seul avion de chasse Rafale. Voir à ce sujet FREDET, Jean-Gabriel et Natacha TATU. "Grands contrats: la fin d'un mirage". Le Nouvel Observateur, $n^{\circ} 2357,7$ janvier 2010.

21

L'accord de coopération est accessible sur le site de I'AMBASSADE DE FRANCE : <http:// ambafrance-eau.org/France_eau/ IMG/19750097.pdf> (consulté en avril 2011).

22

Les rapports d'activités du Louvre sont accessibles sur le site du musée: <http://www.louvre.fr/ media/repository/ressources/ sources/pdf/src_document_54251_ v2_m56577569831216908.pdf> (consulté en janvier 2011).

23

Le site de Cergy-Pontoise, dans le Val-d'Oise, a été retenu pour l'établissement du Centre national de conservation, de restauration et de recherches patrimoniales en Tle-de-France. particulier avec l'implantation d'une antenne de l'Université Paris-IV Sorbonne, ouverte en 2006 à Abou Dhabi, ainsi que d'une antenne des écoles de commerce INSEAD et HEC. Par ailleurs, de nombreuses entreprises françaises sont actives aux Emirats, notamment Total, Thalès, CDF Suez, BNP Paribas. En mai 2009, les médias ont couvert le voyage officiel du président Nicolas Sarkozy à Abou Dhabi, voyage motivé par la signature des derniers accords militaires et commerciaux passés entre les deux pays ${ }^{20}$. Parallèlement, le prince héritier du royaume, le sheikh Mohammed bin Zayed al Nahyan, et le président français ont participé à la cérémonie de lancement du projet Louvre Abou Dabi. Précisons que l'aide à la réalisation du musée d'Abou Dhabi entre dans le cadre de l'Accord de coopération culturelle et technique conclu entre les deux pays le 3 juillet 1975, lequel est dûment rappelé en introduction à l'Accord du 6 mars 2007. L'entente de coopération inclut la formation de professeurs, l'échange de chercheurs, d'experts et d'étudiants, la création d'écoles et d'établissements culturels, l'organisation d'expositions et de manifestations scientifiques, techniques et culturelles, ainsi que l'encouragement du tourisme dans les deux pays ${ }^{21}$.

- Les intérêts pour le Louvre. Tout est résumé par Henri Loyrette dans son "Avant-propos" au Rapport d'activités 2007:

Le Louvre Abou Dhabi [...] engendre des ressources considérables, permettant au Louvre d'envisager sereinement un plan d'investissement de moyen et long terme, intégrant un ensemble de chantiers très nécessaires mais qui n'avaient pas trouvé jusqu'à présent leurs financements. Plus largement, le projet irriguera l'ensemble des musées nationaux partenaires ${ }^{22}$.

Les fonds en provenance d'Abou Dhabi vont permettre d'achever la restauration du pavillon de Flore, ces travaux achevant le projet Grand Louvre tel qu'envisagé en 1981 par François Mitterrand. De ce fait les salles d'un des étages porteront le nom d'une éminence des Emirats. Une autre partie des fonds servira à l'élaboration d'un laboratoire des musées de France, d'un centre d'archives, abritant notamment les réserves des musées du Louvre, d'Orsay, des arts décoratifs et des beaux-arts, ainsi que des ateliers de restauration du Louvre et de Versailles ${ }^{23}$.

En fait, la polémique autour du Louvre Abou Dabi fait ressortir la dichotomie entre la fonction de conservation et de 
recherche du musée, qui est une mission d'ordre théorique et intellectuel, et sa nature politico-économique qui a des exigences d'ordre pragmatique. L'accord signé illustre une adaptation des activités du musée aux prises avec des besoins de financement grandissants face aux pressions du marché de l'art et aux réalités du commerce global. Il illustre aussi la volonté française d'affirmer sa présence, si ce n'est sa prépondérance, au sein d'une économie de la culture qui s'universalise.

\section{Diplomatie et musée}

L'autonomie du Louvre est relative puisque le musée appartient à l'État et son directeur, présentement Henri Loyrette, qui a pris les rênes en 2001, est nommé par le président de la République pour un mandat renouvelable de trois ans ${ }^{24}$. Loyrette, dans un entretien du 29 mars 2007 accordé au quotidien Les Échos, est clair sur le positionnement du Louvre:

Le Louvre a toujours eu un rôle central dans la diplomatie culturelle de la France et a vécu au rythme de la vie politique du pays. Lorsque certains accusent le projet d'Abu Dhabi d'être un projet diplomatique et politique, je dois dire que ce n'est ni nouveau ni choquant. Le Louvre n'est en rien un instrument dans cette affaire, et je trouve tout à fait sain et normal que, en tant que premier musée national, il serve les intérêts de la France et contribue à son rayonnement ${ }^{25}$.

Le rôle du Louvre se définit donc par son statut historique de premier musée créé par la République; à ce titre, il joue un rôle de serviteur de l'État et, au moins tacitement semble-t-il, de leadeur des musées nationaux. Et, en effet, la politique culturelle de la France, qui utilise le musée comme moyen diplomatique pour affirmer son rayonnement et sa présence, n'est pas nouvelle. La diplomatie culturelle, sorte de marketing, de branding politique, a toujours été active; Henri Loyrette le réitère dans un entretien en 2007 :

Le Louvre a été voulu par la Révolution et par l'Empire comme un musée universel rassemblant des œuvres de toutes les époques et de toutes les civilisations, et s'adressant au monde entier. Depuis plus de deux cents ans, il a

\section{4}

Le Conseil des ministres a renouvelé, en 2010, Henri Loyrette dans ses fonctions à la tête du Louvre. II s'agit de son quatrième mandat. 25

DE GASQUET, Pierre et Nathalie SILBERT. "Le Louvre à Abu Dhabi : ambition culturelle ou levier financier ? ". Les Échos. n 19888, 29 mars 2007, p. 8. 


\section{6}

LOYRETTE, Henri. "Nous avons hérité d'une vocation universaliste". L'Humanité, 20 janvier 2007. Accessible sur le site du quotidien: <http://www.humanite.fr/2007-0120_Cultures_Les-musees-doiventils-louer-leurs-oeuvres> (consulté le 16 avril 2010).

27

Voir "Mot du président", disponible sur <www.louvre.fr//llv/musee/ mission.jsp> (consulté en novembre 2010).

28

WALLIS, Brian. "Selling Nations: International Exhibitions and Cultural Diplomacy». In. SHERMAN, Daniel et Irit ROCOFF. Museum Culture: Histories, Discourses, Spectacles. Minneapolis: University or Minnesota Press, coll., "Media G Society", 1994, p. 266.

29

[Traduction libre] BAYLISS, Sarah $\mathrm{H}$. "A Positive Understanding of Islam". ARTnews, nº 5 , mai 2008. "Museums around the world are showcasing Islamic art in an effort to promote understanding and engage immigrant communities." accompagné l'histoire et la diplomatie de notre pays. Les grands départements archéologiques, avec les liens nombreux et durables qu'ils ont tissés avec les pays du bassin méditerranéen du Proche et du Moyen-Orient, sont notamment là pour le rappeler. A l'heure du repli identitaire, cette vocation universelle, héritée du siècle des Lumières et de la Révolution doit être réaffirmée ${ }^{26}$.

Dans un texte de présentation de la mission du musée, Loyrette précise: "Le Louvre, de plus en plus, joue un rôle majeur dans la diplomatie culturelle [...] Cette ouverture internationale se traduit bien sûr par l'organisation d'expositions " ${ }^{27}$. Ces expositions sont donc destinées à "vendre" une certaine image de la France, ou celles d'entités sélectionnées, dans un but stratégique, images fabriquées par le biais du choix des objets et par le discours muséographique mis en place. Les réalités du musée sont donc liées aux contextes politiques intérieurs et extérieurs, ce qui en fait un des acteurs des relations nationales et internationales. Brian Wallis montre comment les instances du pouvoir s'appuient sur le musée pour transformer des perceptions sociales, culturelles ou religieuses, en présentant les communautés par le biais de visions culturelles filtrées, à l'instar des grandes expositions internationales qui sont un des moteurs de la diplomatie globale. Pour cet auteur, il n'y a pas de "mexicanité", d'"américanité" distinctes de celles générées par le déploiement diplomatique par le biais de l'expansion populiste du rôle du musée. Il écrit:

Visual representations are a key element in symbolizing and sustaining national communal bonds. Such representations are not just reactive (that is, depictions of an existing state of being), they are also purposefully creative and they can generate new social and political formations ${ }^{28}$.

Dans cette logique, un article paru en mai 2008 est exemplaire; il a pour sous-titre: "Les grands musées occidentaux mettent en avant l'art islamique afin de promouvoir la compréhension et d'impliquer les communautés immigrées "29. Ces quelques mots insinuent, pour le meilleur ou pour le pire, l'implication de motivations autres que purement scientifiques et désintéressées. Pour illustrer ce point, notons qu'à Paris, après l'exposition Palestine - La création dans tous ses états, le public a pu visiter (jusqu'au 24 mars 2010) l'exposition Arts de l'Islam - chefs-d'œuvre de la collection 
Khalili. Il faut préciser que ces expositions étaient présentées à l'Institut du monde arabe (IMA), fondation créée en 1980 et fruit d'un partenariat entre la France et 24 pays arabes pour faire connaître la culture, favoriser les échanges et resserrer les liens entre l'Europe et le monde arabe. Au demeurant, le Dictionnaire des politiques culturelles françaises fait remonter au Moyen Âge l'origine de l'action publique dans ce domaine: "Dès le XIVe siècle, et plus encore au XVe, on trouve un mécénat royal qui parvient progressivement à étouffer l'action culturelle des Églises, municipalités, corporations et autres corps $"{ }^{30}$. Selon cet ouvrage, le soutien aux créateurs par le droit à "l'exception culturelle" " serait une des dernières manifestations de cette longue tradition de politique interventionniste. C'est d'ailleurs dans le cadre de la politique culturelle que Louis Pasteur, Ferdinand de Lesseps, Jules Verne, Ernest Renan et Armand Colin ont créé, en 1883, l'institution Alliance française, présente aujourd'hui dans plus de 1000 locations éparpillées dans près de 150 pays et qui participe à la promotion de la langue et de la culture françaises dans le monde ${ }^{32}$.

Le musée n'est donc pas un lieu neutre. Tout au long du XIX siècle, il participe à la construction de l'identité nationale; aujourd'hui, il souhaite affirmer sa présence au sein d'une culture qui s'universalise en encourageant le «dialogue entre les cultures ". Toutefois, et fort à propos, l'historien Laurent Gervereau note:

L'ICOM est fondé en 1946 au lendemain de la guerre, pour favoriser "l'éveil et l'enrichissement de la compréhension entre les peuples. " Cette mission [...] est tout à fait à contre courant du grand mouvement constitutif des musées depuis le XIX siècle, à savoir l'illustration sur pièces du nationalisme émergeant et de fortes revendications identitaires ${ }^{33}$.

Le Louvre peut donc se prévaloir de cette nouvelle mission du musée puisque le contrat avec Abou Dhabi prévoit la création d'un musée qui participe au dialogue entre les cultures. L'article $1^{\text {er }}$ de l'accord précise :

Les Parties décident de la création d'un musée universel [...] destiné à œuvrer au dialogue entre l'Orient et l'Occident, chaque Partie respectant les valeurs culturelles de l'autre [...] Les galeries du Musée laissent une large place aux
30

DE WARESQUIEL, Emmanuel (dir.). "Exception culturelle». In. Dictionnaire des politiques culturelles de la France depuis 1959. Paris: Editions Larousse et CNRS, 2001. 31

L'exception culturelle limite le libreéchange en matière de culture afin de protéger le marché artistique national.

32

Voir le site de I'ALLIANCE FRANCAISE: <http://www. alliancefr.org> (consulté en avril 2011).

33

GERVEREAU, Laurent. Vous avez dit musées? Tout savoir sur la crise culturelle. Paris: CNRS, coll. "Carré des sciences", 2006. 
34

BENETON, Philippe. Histoire de mots: culture et civilisation. Paris: Presses de la Fondation nationale des sciences politiques, 1975, p. 34. 35

Cité par BENETON, id., p. 35. confrontations entre des œuvres de périodes et d'aires géographiques différentes, mettant en valeur le dialogue entre les civilisations dans le domaine des Arts [...] Le parcours de visite est organisé selon des thématiques qui offriront au visiteur une vision large et novatrice des enjeux artistiques, centrée sur des genres (le portrait, le paysage...), sur des concepts (le voyage...) ou l'expression visuelle des relations entre l'homme et le monde (l'art funéraire...).

Cette approche dialogique inédite, l'expertise des historiens d'art, le regard des spécialistes, des intellectuels et du public moyen-oriental sur le patrimoine occidental ne peuvent qu'être fertiles en interprétations et bénéfiques à l'histoire de l'art. Dans cette perspective, l'intérêt scientifique, même s'il n'est pas le moteur premier de l'accord, est assez clair. La fonction contemporaine du patrimoine serait-elle de servir la société universelle? Serait-ce alors le nouveau programme idéologique du musée du XXI siècle?

\section{Un musée universel}

$\mathrm{Au} \mathrm{XVIII}{ }^{e}$ siècle, en même temps que les collections princières deviennent publiques et que s'ouvrent les premiers musées modernes, les concepts de civilisation et d'universalisme se développent. Philippe Bénéton écrit que la théologie se séparant de la pensée philosophique, l'histoire humaine devient progressiste, visant l'état de "civilisation-perfection " ${ }^{34}$. L'aplomb du colonisateur est par ailleurs illustré par Georges Gusdorf: "Le siècle des Lumières qui s'enchante de l'idée de Progrès, se voit naturellement en tête du mouvement, dépositaire de la plus haute vérité, missionnaire du nouvel Évangile de la Raison, qu'il devra porter jusqu'aux extrémités de la terre. Tel est le fardeau intellectuel de l'homme blanc " ${ }^{35}$. Le programme idéologique est justifié par les bienfaits que la civilisation universelle est supposée entraîner.

Dans le "Mot du président" daté de septembre 2009, Henri Loyrette déclare, à propos du rayonnement et de l'ouverture internationale du Louvre:

Il s'agit de s'ouvrir à des terres nouvelles, pour couvrir les champs géographiques actuellement absents ou 
sous-représentés dans nos collections. C'est en particulier le cas de l'art des Amériques, de l'art slave, du Soudan ou de l'Asie centrale. Depuis quelques années, le Louvre s'attache ainsi à combler ces manques, que ce soit à travers sa politique d'acquisitions et d'expositions, ou encore par le biais d'accords de coopérations culturelle et scientifique ${ }^{36}$.

Si le musée universel occidental continue d'acquérir, de se rendre "propriétaire " d'œuvres de régions éloignées, pour contribuer à une meilleure compréhension du monde et au rayonnement international du musée comme l'avoue Loyrette, il prive par là même les cultures locales de leur droit de se représenter elles-mêmes, de déterminer ellesmêmes le destin de leur propre héritage. C'est aussi ce que remarque Andrew McClellan quand il demande si la notion de l'intérêt de la postérité universelle justifie le transfert des objets et si elle est plus importante que le respect du contexte des objets, du respect de la mémoire et des cultures locales ${ }^{37}$.

Le concept d'universalité a donc pour centre l'Europe, et le musée est universel à deux points de vue. D’abord parce qu'il cherche à rassembler tous les symboles matériels de tous les temps et de tout le monde. Et pour accomplir cette tâche, au demeurant impossible, de vouloir tout posséder, tout classifier et tout étiqueter, il se targue de sa mission civilisatrice à l'échelle universelle. Le musée est l'instrument civilisateur par excellence, aussi bien en son pays que partout ailleurs.

L'élaboration du Louvre Abou Dabi est une création sans rapport avec l'histoire de la région. Il ne sera pas pour autant le premier dans la région. Pour ne citer que quelques-uns des derniers venus, parmi les plus prestigieux: le Musée national d'Arabie saoudite ouvert en 1999 à Riyad; le Musée d'art islamique de Doha au Qatar ouvert en 2008, qui doit être suivi par une dizaine de musées au cours de la prochaine décennie, dont le Musée national du Qatar; le Musée de la civilisation islamique ouvert en 2008, à la suite des musées (archéologie, science, planétarium, etc.) ouverts au cours des dernières années dans l'émirat de Charjah des ÉAU ${ }^{38}$; le Musée national du royaume
LOYRETTE, Henri. "Mot du président ". Accessible sur le site Internet du Louvre, op. cit. 37 MCCLELLAN, Andrew. The Art Museum from Boullée to Bilbao. Berkeley (CA), Los Angeles et Londres: University of California Press, 2008, p. 233-234. 38

En 1998, l'UNESCO a proclamé Charjah la capitale culturelle du monde arabe. 
39

CLIFFORD, James. Routes. Travel and Translation in the Late Twentieth Century. Cambridge (MA) et Londres: Harvard University Press, 1997. p. 188-219.

40

Id., p. 210. de Bahreïn ouvert en 2009 à Manama. La particularité du musée d'Abou Dhabi est qu'il sera le premier musée «universel" hors Occident.

Pour l'historien et anthropologue James Clifford ${ }^{39}$, un musée ne peut pas être universel puisqu'il est impossible d'exposer de manière fiable et figée une quelconque culture, d'abord parce que celle-ci est sujette à d'infinies interprétations et parce que la mise en représentation implique des relations de pouvoir, des rapports hiérarchiques de dominants (le centre politique et social, lieu de rassemblement des objets) et de dominés (la périphérie mise en scène et en spectacle) qui faussent le discours. Clifford préconise, pour éviter l'exposition passive, stéréotypée et sous-tendue par la condescendance patriarcale, l'approche interactive, conjecturale et toujours renouvelée afin que les stratégies d'exposition, de représentation, résultent des interactions particulières de hiérarchie, d'entente, de résistance, de négociations, de réciprocité, dans un lieu et à un moment précis. Cette stratégie muséale implique la multiplication des contacts entre spécialistes, scientifiques et praticiens, car aucune de ces personnes n'a l'expertise et l'expérience, sans le recours aux autres, pour contextualiser et "faire parler" les collections. Par ailleurs, elle implique le redéploiement constant des collections en fonction des réappropriations effectuées à l'issue de ces échanges. Clifford écrit :

The fact that an altar or a tribal mask can mean quite different things in different locations makes inescapable the recognition and display of multiple contexts for works of art or culture. Innovative museums professionals have long been interested in ways to put objects in a fresh light, to make them new. Explicit contact relations now place this kind of search in a different conjuncture, imposing new collaborations and alliances ${ }^{40}$.

Le musée du XIX ${ }^{e}$ siècle qui servait à édifier et à éduquer le public devrait aujourd'hui se transformer, selon la prescription de Clifford, en un lieu où les objets ne racontent pas une histoire mais des histoires, en un lieu de rencontres interculturelles, un lieu d'interprétions ponctuelles, de discours assumés, de débats et de critiques. La méthode permettrait, sans évidemment pouvoir les abolir, de dépasser le stade des relations de pouvoir, de dépasser la 
représentation "exotique" de l'Autre, de bousculer centre et périphérie en impliquant toujours de nouveaux joueurs pour interpréter des objets et les mettre en scène; elle permettrait de dépasser le stade du pseudo-musée neutre pour assumer, afficher et jouer de la partialité du musée. Cette stratégie, selon Clifford, est applicable aux petites institutions comme aux grands musées universels :

What would it take (and why would it matter?) to treat the Metropolitan Museum of Art in Manhattan as a contact zone rather than a center? Or the Louvre? To give marginal "between" places a tactical centrality is ultimately to undermine the very notion of a center. All sites of collection begin to seem like places of encounter and passage. Seen this way, objects currently in the great museums are travelers, crosserssome strongly "diasporic" with powerful, still very meaningful, ties elsewhere. Moreover, the "major" museums increasingly organize themselves according to the dictates of tourism, national and international. This rethinking of collections and displays as unfinished historical processes of travel, of crossing and recrossing, changes one's conception of patrimony and public ${ }^{4}$.

Le musée comme "zone de contact» est dynamique, inclusif, dialogique, créateur de nouvelles collaborations et de nouveaux discours. Dans cette perspective, le Louvre Abou Dabi trouverait alors sa pleine justification et sa pleine valeur.

Évidemment, le respect de la diversité culturelle envisagée selon les principes de la probité, de la réciprocité des collaborations et des échanges, de la circulation d'expositions, entraîne la question de l'atteinte à l'intégrité des œuvres et bouleverse le rôle inaugural du musée du Louvre, musée encyclopédique aspirant à l'exhaustivité des collections en un lieu unique pour consultation intégrale.

\section{Le statut de patrimoine national est-il à revoir ?}

La polémique autour du Louvre Abou Dabi montre la valeur culturelle accordée au patrimoine sous réserve toutefois que celui-ci soit maintenu hors du circuit économique. Le sociologue Henri-Pierre Jeudy remarque que le défi contemporain réside dans la gestion du patrimoine qui doit avoir un statut "à part", retiré du marché pour conserver sa valeur symbolique mais, et c'est là selon lui le dilemme, "il n'y a pas de développement culturel sans 
42 JEUDY, Henri-Pierre. La Machinerie patrimoniale. Belval (Vosges): Circé, 2008, p. 16.

43

"Les musées français à l'heure d'Abou Dhabi. Entretien avec Françoise Cachin et Krzysztof Pomian ". Le Débat, n 145 , mai-août 2007.

44

Pour certains, le multiculturalisme dénonce l'hégémonie occidentale et demande la reconnaissance et l'acception politique et sociale de toutes les minorités. commercialisation [...] Les stratégies les plus courantes désormais s'orientent vers une combinaison qui contient cette contradiction : ce qui est tenu pour sacré n'empêche pas la circulation des valeurs matérielles " ${ }^{42}$. En effet, avec le dossier Abou Dhabi, les deux objectifs cherchent à cohabiter, mais cela ne se fait pas sans heurts. C'est d'ailleurs dans ce même esprit que Krzysztof Pomian, pour qui l'idée de retrait des objets symboliques de la sphère économique est au centre de sa recherche, admet:

L'époque où la culture était soustraite par définition au fonctionnement des règles économiques est révolue. C'est un fait que nous devons prendre en compte [...] Cela se répercute dans les musées qui fonctionnaient alors dans le cadre d'une économie du don et du prestige et non pas dans celui d'une économie marchande. Il est à craindre que l'espace de l'économie du don soit aujourd'hui très fortement rétréci, que la culture soit entrée pour une part beaucoup plus grande qu'avant dans la sphère des relations marchandes et que la vraie question qui se pose est de savoir comment nous devons réagir ${ }^{43}$.

Certes, le projet Louvre Abou Dabi semble être un des garants de la poursuite du commerce entre la France et les Émirats arabes unis; il est source d'importants revenus pour les musées français par le biais de tractations contractuelles qui réintroduisent en quelque sorte les collections patrimoniales dans le circuit économique.

Par ailleurs, le cosmopolitisme des sociétés contemporaines entraîne une certaine révision du concept de l'État national basé sur la cohésion identitaire de la population. Aujourd'hui, et même si le terme reste sujet à débats ${ }^{44}$, le multiculturalisme distingue les différents groupes qui coexistent dans une société, ou dans un pays, et cherche à leur donner une place équitable. Le philosophe Patrick Savidan le définit ainsi :

Le multiculturalisme est une conception de l'intégration établissant qu'il est en quelque sorte du devoir de l'État démocratique de "reconnaître", d'une part, la multiplicité des groupes ethnoculturels qui composent de manière significative sa population, et de chercher, d'autre part, à accommoder dans la mesure du possible, sur la base de principes clairement identifiables, cette diversité 
culturelle. La conception multiculturaliste marque par conséquent le renoncement à un point de vue assimilationniste fort qui se fonde sur un principe de stricte indifférenciation dans la reconnaissance ${ }^{45}$.

Cet auteur précise que le multiculturalisme est aujourd'hui l'objet d'une certaine reconnaissance politique et sociale qui, à terme, peut transformer le monde géopolitique en faisant reculer l'idée d' Etat-nation" construit autour d'une appartenance ethnoculturelle dominante, laquelle nivelle les autres groupes présents au sein de chaque État. Savidan appuie son raisonnement sur les théories du philosophe Jürgen Habermas, qui considère qu'une des solutions pour dépasser la crise politique, sociale et culturelle des États modernes doit reposer sur une conception "postnationale" de l'État. En effet, dans son ouvrage Après l'Étatnation, Habermas développe le concept de "patriotisme constitutionnel" qui devrait remplacer le "patriotisme national" basé sur une communauté ethnique prépondérante partageant la même histoire. Selon lui, le patriotisme constitutionnel permet de rallier, sans les assimiler, les différents groupes culturels d'un territoire, derrière des valeurs juridiques et sociales démocratiques communes, acceptées et respectées par tous; il permet de transformer l'«État-nation" en "État-social" engendrant la création d'une "citoyenneté multiculturelle", recomposant ainsi la base de la solidarité de la population ${ }^{46}$. Habermas affirme en outre que la mondialisation de l'économie affecte le fonctionnement et la légitimité des États-nations, que le politique doit donc s'adapter et penser au-delà de l'Étatnation historique pour envisager le gouvernement d'un État-social sous l'égide d'un système politique "supranational" assurant une certaine équité et une solidarité "cosmopolitique " ${ }^{47}$. C'est ce que résume Savidan quand il écrit que "la conception 'postnationale' de l'État doit [...] permettre à la politique de se hisser au niveau de l'économie mondiale, en ne demeurant plus confinée dans les limites devenues trop étroites des nations ${ }^{48}$. Et si tel est le cas, le patrimoine national devient, par extension, un concept également trop étriqué pour représenter la multiplicité des identités des ressortissants d'un pays et l'idée d'un patrimoine "postnational" devient du même coup tout aussi
SAVIDAN, Patrick. Le multiculturalisme. Paris: Presses universitaires de France, coll. "Que sais-je?", 2009, p. 17.

46

HABERMAS, Jürgen. Après l'Etatnation. Une nouvelle constellation politique. Paris: Fayard, 2000, p. 66-70.

47

Id., p. 33-37.

48

SAVIDAN, op. cit., p. 7. 
49

Un neuvième département est en cours de conception. Il sera consacré aux arts des chrétientés d'Orient, des empires byzantins et slaves. Voir FLOUQUET, Sophie. "Musée du Louvre. Vers un $9^{e}$ département". Le Journal des Arts, n 317, 22 janvier 2010, p. 36.

50

Dernier département patrimonial du Louvre créé par décret du $1^{\mathrm{er}}$ août 2003. Il doit ouvrir au public en 2012. 51

CUNO, James. Who Owns Antiquity? Museums and the Battle over Our Ancient Heritage. Princeton (NJ): Princeton University Press, 2008, p. 123: "[Encyclopedic art museums] bear witness to the hybridity and interrelatedness of the world's cultures." plausible, car il pourrait alors refléter la diversité culturelle et les modes d'appartenance non nationalistes.

Par ailleurs, peut-on affirmer que les collections du Louvre sont le juste héritage des biens transmis par les ancêtres de la nation, sachant que la majorité des œuvres abritées dans le musée ne sont pas d'artistes français, ainsi la Joconde, la Vénus de Milo, la Victoire de Samothrace pour ne citer que ces trois stars? Même si elles font partie de l'héritage historique du musée, ne font-elles pas plus justement partie du patrimoine de l'humanité ? Le musée du Louvre rassemble 35000 objets, sur plus de 60 ooo mètres carrés de salles d'expositions permanentes. Ces objets sont aujourd'hui répartis en huit départements ${ }^{49}$, nommément: les Antiquités orientales, les Antiquités égyptiennes, les Antiquités grecques, étrusques et romaines, les Arts de l'Islam ${ }^{50}$, les Sculptures, les Objets d'art, les Peintures et les Arts graphiques. La diversité du pouvoir créatif du monde entier est, plus ou moins, représentée dans le musée. Dès lors, la visée identitaire mondiale est plus compréhensible que la visée identitaire nationale, car les collections du Louvre permettent à tout visiteur de retrouver la ou les cultures dont il se réclame en ayant accès aux univers artistiques de presque toute la planète avec, en plus, la possibilité de tisser de nouveaux liens selon ses affinités personnelles. Les musées de nature encyclopédique, souligne James Cuno, témoignent de l'hybridité et des corrélations qui existent entre les cultures du mondes'. Ils permettent d'élargir les horizons de chacun et d'avoir accès à l'intemporalité' et à l'universalité de l'art, c'est-à-dire à une vérité qui va au-delà des considérations géographiques, politiques et culturelles. Incidemment, une œuvre conservée dans le département des Antiquités grecques, étrusques et romaines peut provenir d'un territoire national dépendant d'un autre département du Louvre, comme le département des Antiquités orientales, à l'instar de la mosaïque grecque Le Jugement de Pâris, réalisée vers l'an 115 de notre ère et provenant de la ville d'Antioche, aujourd'hui située en Turquie orientale et rebaptisée Antakya. Cette situation, entre autres, appuie la thèse de Cuno qui soutient que les antiquités ne peuvent appartenir à une juridiction particulière, 
car ce sont les hasards de l'Histoire qui les font se trouver sur le territoire des États, des nations modernes. Pour cet auteur, c'est l'intérêt du public universel qui doit dicter aux musées, et derrière eux aux nations, les politiques de conservation, d'accès au savoir et d'accès aux œuvres ; il suggère que l'attention se détourne de la "propriété des œuvres" pour s'orienter vers le gardiennage et l'administration des œuvres, car, peu importe leur propriétaire, elles sont dans les collections du musée non pas pour le musée lui-même, mais pour le public qu'il sert ${ }^{52}$. Les œuvres sont l'héritage historique commun; elles sont sous la tutelle des musées dans la mesure où ceux-ci conservent la confiance du public, qu'il soit local ou global.

Près de $70 \%$ des visiteurs du Louvre sont des visiteurs en provenance de l'étranger; le musée attire donc les "nationaux" aussi bien que les "étrangers", quelles que soient les raisons de leur visite. Et au-delà des chiffres et des statistiques, la mondialisation fait graduellement prendre conscience qu'il n'y a pas de patrimoine ou d'entité, comme la nation ou l'État, qui puisse représenter chacun des habitants d'un pays donné. En effet, les mouvements migratoires, la conception pluraliste, les marchés mondialisés, les nouveaux modes de communication, changent les paramètres historiques en multipliant le flux des contacts et des influences qui favorisent l'émergence d'une identité personnelle, et collective plurielles. Le besoin d'appartenance est sans doute une aspiration légitime, mais c'est en reconnaissant les nombreuses affiliations de chacun que l'on peut espérer enrayer les dérives communautaristes ou intégristes. L'essayiste Amin Maalouf écrit à ce sujet :

Chacun d'entre nous devrait être encouragé à assumer sa propre diversité, à concevoir son identité comme étant la somme de ses diverses appartenances, au lieu de la confondre avec une seule, érigée en appartenance suprême, et en instrument d'exclusion, parfois en instrument de guerre [...] De la même manière, les sociétés devraient assumer, elles aussi, les appartenances multiples qui ont forgé leur identité à travers l'Histoire, et qui la cisèlent encore; elles devraient faire l'effort de montrer, à travers des symboles visibles, qu'elles assument leur diversité, afin que chacun
52

Id., p. 13: "Does it really matter who owns a particular antiquity - whether it is a museum in the first world or a nation in the third world? Museums own antiquities (and all works of art in their collections) only insofar as they hold them in trust for the public they serve." 
53

MAALOUF, Amin. Les identités meurtrières. Paris: Grasset, 1998, p. $183-184$.

54

Id., p. 143 puisse s'identifier à ce qu'il voit autour de lui, que chacun puisse se reconnaître dans l'image du pays où il vit, et se sente encouragé à s'y impliquer plutôt que de demeurer, comme c'est trop souvent le cas, un spectateur inquiet, et quelquefois hostile ${ }^{53}$.

Dans cette perspective, le Louvre Abou Dabi ne contribue-til pas à faire émerger et respecter une culture plurielle universelle? C'est en tout cas la position de ceux qui défendent le projet, mais Maalouf tempère quelque peu l'utopisme sous-jacent, par cette réflexion:

Il serait désastreux que la mondialisation [...] fonctionne à sens unique, d'un côté les "émetteurs universels", de l'autre les "récepteurs"; d'un côté "la norme", de l'autre "les exceptions"; d'un côté ceux qui sont convaincus que le reste $\mathrm{du}$ monde ne peut rien leur apprendre, de l'autre ceux qui sont persuadés que le monde ne voudra jamais les écouter ${ }^{54}$.

Le Musée national du Grand Louvre, héritage des Lumières, gardien du patrimoine, vecteur d'identité, reste aujourd'hui une institution nationale, un lieu de pouvoir et d'économie marchande. Peut-on alors soupçonner la France de tentation hégémonique, et les Émirats arabes unis d'être victimes d'une nouvelle forme de l'impérialisme français? Dans le principe, la réponse est négative puisque c'est le gouvernement émirien qui est le demandeur et le payeur, en un mot le "client", tandis que le gouvernement français est son obligé, le pourvoyeur. Dans les faits, qui sont plus insidieux, la réponse ne peut qu'être nuancée; elle incite à une réflexion construite à partir des études postcoloniales afin de pouvoir analyser, et mettre à jour, l'éventuelle persistance des rouages historiques de l'eurocentrisme et de l'impérialisme culturel. Dans cette perspective, on peut soupçonner Abou Dhabi, financièrement très riche, de désirer "acheter" un musée occidental, d'acquérir par là même la crédibilité et le prestige occidentaux.

\section{Figures prospectives}

Le philosophe Bernard Deloche rappelle que le patrimoine est d'abord de nature privée, qu'il se transmet pour être exploité socio-économiquement. Par contre, le patrimoine, héritage public non économique, qui n'existe que métaphoriquement, bâtit sa justification, sa "valeur morale" 
et sa réputation sur l'idéologie nationale qu'il contribue à fonder :

Le seul patrimoine que connaisse effectivement la société capitaliste est un patrimoine économique et privé, l'autre n'est qu'une fiction, comme l'ont fort bien montré Pierre Bourdieu et Alain Darbel dans leur étude sociologique de la fréquentation des musées ${ }^{55}$.

Aujourd'hui, les expositions du musée sont élaborées pour stimuler l'intérêt, ou au moins la curiosité, et activer l'image de la culture française dans le monde, mais tout porte à croire que le musée doive non seulement stimuler la narration d'un discours identitaire pour assurer sa raison d'être, mais qu'il doive, en même temps, initier des approches mercantiles nécessaires à l'élaboration de ses projets culturels et scientifiques. Et cela semble devoir passer par la rentabilisation du capital. Ce patrimoine collectif moral, fruit d'une construction culturelle, rejoint-il finalement le patrimoine privé, dans sa quête de capitalisation économique?

Deloche analyse le patrimoine du musée en remontant à son origine théorique et historique. Dans un essai de 2002, Andrew McClellan analyse, quant à lui, la place du musée dans la société. Il déduit que celui-ci ne peut se réduire à quelques métaphores telles que "temple", "tombeau", ou "centre d'achats", car elles ne tiennent pas compte de l'espace utopique qu'il représente. Selon lui, le musée répond à la quête humaine de la perfection et de la connaissance; il est le témoin de la créativité et de la valeur individuelles; il est porteur de valeurs intemporelles et d'aspirations sociales. Et c'est par sa capacité à participer et à nourrir les rêves de la société qu'il intéresse et qu'il devient un outil des gouvernements, des entreprises commerciales, des philanthropes:

If respite from the modern world and engine of urban renewal are new ideals projected onto our museums, they continue to be a source of nourishment for the dream of a unified, harmonious society, which has acquired added urgency in recent decades in step with increased multicultural awareness. Witness, for example, the mission statement for the new de Young Museum in San Francisco, to be designed by Herzog, and de Meuron. Not only does the museum promise to be an "urban oasis" contributing to "the City's crusade for urban renewal," but it will serve
55

DELOCHE, Bernard. Museologica. Contradictions et logique du musée. Paris: Editions J. Vrin, coll. "Science, Histoire, Philosophie", 1985, p. 51-52. 
56

MCCLELLAN, Andrew. "From Boullée to Bilbao. The Museum as Utopian Space". In. MANSFIELD, Elizabeth (dir.). Art History And Its Institutions. Foundations of a Discipline. Londres et New York: Routledge, 2002, p. 62. as "a common ground where-through art-the usual boundaries that separate us from each other: culture, creed, race, and all the others, become bridges that connect us"56.

Ainsi, le discours muséal est fondé sur une promesse et, même si les enjeux culturels de la mondialisation sont énormes, sa contribution à une meilleure compréhension du monde semble être une motivation collective essentielle. Dans cette perspective, le musée peut devenir une sorte de centre culturel, de centre d'interprétation du patrimoine, sorte de lieu de rencontres des œuvres et des publics, de lieu de débats et, pourquoi pas, de contestations. Les œuvres des collections sont alors appelées à bouger, au sein même du musée, à être re-présentées, pour renouveler le dialogue des œuvres entre elles et entre les publics, mais également à voyager, à aller à l'encontre de nouveaux rapprochements d'œuvres, de nouveaux échanges de propos, de nouveaux discours féconds.

Au XIX $\mathrm{X}^{\mathrm{e}}$ siècle, le musée du Louvre se présente comme un dispositif qui participe à la fabrication de la cohésion nationale; il sert le pouvoir et représente l'arrimage culturel que celui-ci propose. Au XXe siècle, il se développe, se démocratise et se modernise. Au XXI siècle commençant, il cherche à s'adapter aux nouvelles données du temps en mettant en place de nouvelles règles d'administration, notamment suivant l'Accord Louvre Abou Dabi. Le Louvre Abou Dabi, après la polémique suscitée lors de son élaboration, s'avérera-t-il un musée prospère, non pas seulement économiquement, mais tel que mis en avant par ses promoteurs, en tant que "musée-pont", promouvant l'intérêt, le dialogue, l'entente et le respect entre les cultures, entre l'Orient et l'Occident? Rappelons que le futur musée émirien n'est pas conçu pour être un musée "satellite" ou une branche du musée parisien. Les prestations, les prêts d'œuvres sont prévus pour une durée de dix ans, l'envoi des expositions annuelles pour une durée de quinze ans, et ce, à partir de l'ouverture du musée d'Abou Dhabi ; le prêt du nom "Louvre" est prévu pour une durée de trente ans et six mois à partir de la signature de l'accord. Si le musée émirien ouvre ses portes, comme anticipé, en 2014, le prêt d'œuvres cessera en 2024, l'envoi des expositions 
temporaires contractuelles en 2029, et en septembre 2037 le musée devra abandonner l'appellation "Louvre". Il sera alors un musée de nature encyclopédique, si ce n'est un musée "universel", doté exclusivement des collections que les Émirats auront constituées, notamment avec l'aide des experts français. Quel rôle jouera alors ce nouveau musée, aussi bien sur la scène nationale, que sur la scène internationale? 
Summary

Translated by Olga Corban

The Louvre Abu Dhabi:

a Dialogue between Cultures or the Marketing of Cultural Heritage 
This article asks the question: What is the ideological meaning of the Intergovernmental Agreement signed as of March 5 , 2007, which currently assists France in establishing the first universal museum outside the Occident, in Abu Dhabi, the United Arab Emirates? Some of the issues raised include: interference in the affairs of the museum; the marketing of the name "Louvre"; and likewise, of works of national cultural heritage. The article also analyses the historical role of the State museum in society, the edification of the notion of national cultural heritage and its relevance today as it is faced with the cosmopolitanism of modern societies, pluralism and globalization. It shows that the economic resources of the Louvre and its methods to create the necessary funds to maintain the balance in the budget, thus growing the financial profit of national public museums and underlines the wish to impose the French presence within a culture that is becoming universal.

The signed Intergovernmental Agreement is witness to the adaptability of the national museum to meet growing financial needs in the context of an increasing pressure from art market and the realities of global trade and the merchant economy of the culture. The agreement also illustrates the opposition between pragmatic requirements of the museum's political-economic direction and the theoretical and moral functions of conservation and research. It also shows that the museum's symbolic capital is also financial. 